\section{Eosinophile Entzündung spielt auch bei mildem persistierenden Asthma eine Rolle}

Ferguson GT et al. Benralizumab for patients with mild to moderate, persistent asthma (BISE): a randomised, double-blind, placebo-controlled, phase 3 trial. Lancet Respir Med 2017; 5: 568 576. doi:10.1016/S2213-2600(17)30190-X

Bei schwerem eosinophilen Asthma können mit einer gegen den Interleukin-5(IL-5)-Rezeptor- $\alpha$ gerichteten Therapie die Eosinophilen depletiert werden. Asthmaexazerbationen verringern sich, die Lungenfunktion wird verbessert. Auch bei mildem bis moderatem persistierenden Asthma treten erhöhte Interleukin-5-Serumkonzentrationen als Zeichen einer eosinophilen Entzündung auf.

Eine randomisierte Doppelblind-Studie prüfte deshalb Wirksamkeit und Sicherheit des anti-IL-5-Rezeptor- $\alpha$-Antikörpers Benralizumab bei mildem bis moderatem persistierenden Asthma im Vergleich zu Placebo. Die zwischen 18 und 75 Jahre alten Patienten nahmen bei Rekrutierung niedrige bis mittlere Dosierungen von ICS oder eine Fixkombination von niedrig dosiertem ICS plus langwirksamem Betamimetikum (LABA) ein. Unter dieser Therapie hatten sie in der letzten Woche vor Randomisierung am Tag oder nachts an mindestens zwei Tagen an Symptomen gelitten oder für mindestens zwei Tage ein Bedarfsspray einge- setzt oder waren in mindestens einer Nacht durch das Asthma aufgewacht. Die Einsekundenkapazität (FEV1) nach Inhalation eines Bronchodilatators musste mindestens zu $12 \%$ reversibel sein, der FEV1-Wert am Morgen vor Bronchodilatation zwischen 50 und $90 \%$ vom Soll entsprechen. Gary T. Ferguson vom Lungenforschungsinstitut Südostmichigan in den USA und Kollegen standardisierten zunächst die ICS-Medikation auf $180 \mu \mathrm{g}$ oder $200 \mu \mathrm{g}$ Budesonid im Trockenpulverinhalator zweimal täglich je nach zugelassener Dosierung im Land des jeweiligen Studienzentrums. Stratifiziert nach Bluteosinophilenzahl und Land erhielten 211 Studienteilnehmer dann randomisiert subkutan entweder Placebo $(n=105)$ oder Benralizumab (30 mg, $\mathrm{n}=106$ ) alle vier Wochen für insgesamt 12 Wochen. Primärer Endpunkt der Studie war die Veränderung des FEV1 vor Bronchodilatation vom Ausgangwert bis zu Woche 12.

\section{Ergebnisse}

Benralizumab führte zu einem um $80 \mathrm{ml}$ gegenüber Placebo verbesserten Präbronchodilatations-FEV1 als Placebo nach 12 Wochen. Absolut änderte sich der FEV1 in der Placebogruppe kaum von $2.246 \mathrm{ml}$ auf $2.261 \mathrm{ml}$, in der Benralizumab-Gruppe etwas mehr von $2.248 \mathrm{ml}$ auf $2.310 \mathrm{ml}$. Damit wurde allerdings die Grenze dessen, was als klinisch relevant eingestuft wurde (10\%), unterschritten. Die Rate der unerwünschten Ereignisse war in beiden Studiengruppen vergleichbar (Benralizumab: 44\%, Placebo: 47\%).
FAZIT

Nach Meinung der Autoren belegen die Ergebnisse, dass bei Patienten mit mildem bis moderatem persistierenden Asthma auch unter ICS-Therapie entzündliche Krankheitsprozesse fortschreiten können. Die gegen die eosinophile Entzündung gerichtete Antikörpertherapie mit Benralizumab führte zu einer Verbesserung der Lungenfunktion, die zwar nicht klinisch relevant war, aber doch Anlass geben könnte zu weiteren Studien zur Rolle des Antikörpers zur Beeinflussung des Krankheitsprogresses.

Friederike Klein, München 